\title{
The Effect of Foot Compliance Encoded in the Windlass Mechanism on the Energetics of Human Walking
}

\author{
Seungmoon Song ${ }^{1}$, Christopher LaMontagna ${ }^{2}$, Steven H. Collins ${ }^{2}$, and Hartmut Geyer ${ }^{1}$
}

\begin{abstract}
The human foot, which is the part of the body that interacts with the environment during locomotion, consists of rich biomechanical design. One of the unique designs of human feet is the windlass mechanism. In a previous simulation study, we found that the windlass mechanism seems to improve the energy efficiency of walking. To better understand the origin of this efficiency, we here conduct both simulation and experimental studies exploring the influence of foot compliance, which is one of the functionalities that the windlass mechanism embeds, on the energetics of walking. The studies show that walking with compliant feet incurs more energetic costs than walking with stiff feet. The preliminary results suggest that the energy saved by introducing the windlass mechanism does not originate from the compliance it embeds. We speculate that the energy savings of the windlass mechanism are related more to its contribution to reducing the effective foot length in swing than to providing compliance in stance.
\end{abstract}

\section{INTRODUCTION}

Ideally, prosthetic legs should restore or surpass the functionality of that of intact human limbs, substantially improving the quality of life for amputees. Many groups in industry and academia are investing in related research to achieve this goal [1]-[6]. However, few of the studies investigate the potential benefits of the rich biomechanical design of human feet [7], [8], the body part that mostly interacts with the environment during locomotion.

One of the unique features of human feet is the windlass mechanism. The windlass mechanism engages the longitudinal foot arch and the toe segment by the plantar fascia (Fig. 1-a), a thick tendon that spans from the underside of the heel to the toe [9], [10]. Previous biomechanical studies on the windlass mechanism focus on its functionality of passively articulating the toe segment [11]-[13]. Another functionality of the windlass mechanism is to modulate the stiffness of the foot depending on the load the foot is bearing. While the foot segment is flexible at normal configuration (marked in gray in Fig. 1-b), the foot stiffens as it bears weight and the plantar fascia gets loaded (marked in black in Fig. 1-b).

In a previous simulation study [14], we investigated the windlass mechanism's potential of improving the energy efficiency of walking. We showed that walking with feet that incorporate the windlass mechanism could save more than $15 \%$ of the energetic cost as compared to walking without the

\footnotetext{
${ }^{1} \mathrm{~S}$. Song and H. Geyer are with the Robotics Institute, Carnegie Mellon University, 5000 Forbes Avenue, Pittsburgh, PA 15213, USA. \{smsong,hgeyer\} at cs.cmu.edu

${ }^{2}$ C. LaMontagna and S.H. Collins are with the Department of Mechanical Engineering, Carnegie Mellon University, 5000 Forbes Avenue, Pittsburgh, PA 15213, USA. \{cjlamont, stevecollins $\}$ at cmu.edu
}

mechanism. We hypothesized that the energy saving comes either from the foot compliance introduced by the windlass mechanism or from its property of reducing the effective foot length in swing.

In this paper, we investigate the first option: Do compliant feet improve the energy efficiency of walking? To address this question, we develop a foot model, which allows to vary compliance independent of foot length, and test the influence of foot compliance on energy efficiency through simulation and experimental studies (section II and III, respectively). Our preliminary results show that compliant feet actually worsen the energy efficiency of walking (section IV). This suggests that the energy saving of the windlass mechanism originates rather from the foot length changing between stance and swing (section V).

\section{SIMULATION EXPERIMENT OF HUMAN WALKING WITH COMPLIANT FEET}

\section{A. Neuromuscular Human Walking Model}

We used a forward dynamic simulation model of the human musculoskeletal system and its neural control [14], [15] (Fig. 2). The model generates steady walking behavior with human-like kinematics, kinetics, and muscle activations. The musculoskeletal model is planar and consists of the trunk, thighs, shanks and feet segments, which are connected by hip, knee and ankle revolute joints. The joints are actuated by seven Hill-type muscle models per leg, five of which are monoarticular muscles (soleus, SOL; tibialis anterior, TA; vastii group, VAS; gluteus maximus, GLU; and grouped hip flexors, HFL) and two of which are biarticular ones (gastrocnemius, GAS and hamstring group, HAM). The contractile elements of the muscle models take stimulation signals between 0 and 1 , and each muscle force produces (a)

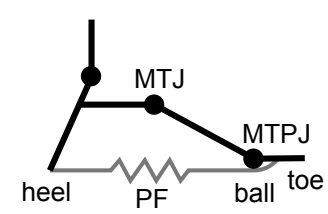

(b)

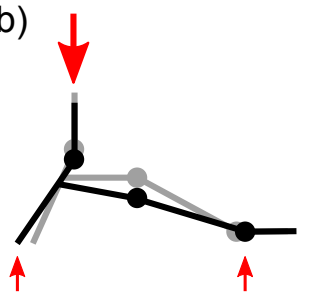

Fig. 1. The windlass mechanism of human feet. (a) Key components of the windlass mechanism: the plantar fascia $(\mathrm{PF})$, which wraps around the ball and connects the heel to the toe; the midtarsal joint (MTJ); the metatarsal phalangeal joint (MTPJ); and the toe segment. When the foot is loaded (red arrows), the windlass mechanism keeps the foot arch from collapsing by pulling the heel toward the ball as shown in (b). 


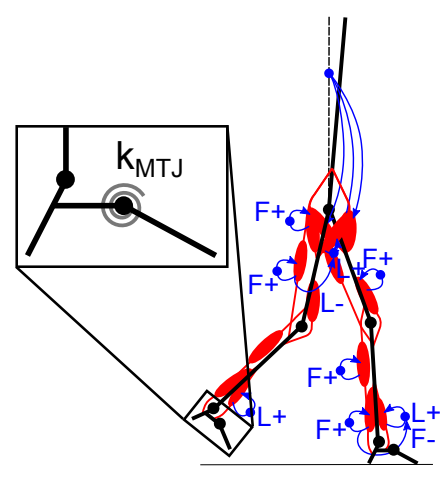

Fig. 2. Neuromuscular human walking model with compliant feet. The stiffness of the foot is modeled by a linear torsion spring $k_{M T J}$ at the midtarsal joint. The blue lines depict modeled reflex pathways (see [15] for more details).

joint torque as $\tau_{m, j}=F_{m} r_{m}\left(\varphi_{j}\right)$ at the joint $j$ it spans, where $r_{m}\left(\varphi_{j}\right)$ mimics variable moment arms observed in physiology. The ground contact and joint limits are modeled as nonlinear spring-dampers. The muscle stimulations are the outputs of the neural control model, which consists of separate stance and swing phase reflexes based on sensory signals measuring muscle states (mostly local positive force or length feedbacks, $F+$ or $L+$, Fig. 2). These sensory and stimulation signals are time-delayed, to model neural transport delays. (See [15] for more details on this model.)

\section{B. A Model of Compliant Feet}

The neuromuscular human model is implemented in MatLab Simulink/SimMechanics (R2012a) using the ode15s solver. We replace the original rigid segment feet with compliant foot models as shown in Fig. 2. Each compliant foot model consists of two rigid segments; the front segment originates at the ball and connects to a second, back segment through the midtarsal joint (MTJ). The latter segment includes the heel and connects to the shank through the ankle joint. The MTJ is passively actuated by a linear torsion spring $k_{M T J}$. The distance between the heel and ball is $20 \mathrm{~cm}$, the height of the ankle joint is $8 \mathrm{~cm}$ from the ground, and each foot weighs $1.25 \mathrm{~kg}$. All these parameters were matched to the dimensions of the human foot.

\section{Energetic Cost Optimization of the Neural Control}

To compare the effect of the foot compliance on energy consumption of walking in simulation, we use an optimization-based approach. We use covariance matrix adaptation evolution strategy (CMA-ES, [16], [17]), to find the optimal control gains that consume minimum energy for each a range of stiffness values $k_{M T J}$. We optimize all 30 control parameters and 7 initial conditions (the initial forward velocity and 6 joint angles). Our implementation of CMA-ES samples 64 sets of the parameters based on a covariance matrix, runs individual simulations to calculate corresponding values of the cost function, and uses the best 32 sets to adapt the covariance matrix to resample new parameters in the next generation. This procedure repeats for hundreds of generations until the optimization does not find a better solution over the last 400 generations.

The cost function that the optimization minimizes is

$$
J_{\text {steady }}=c_{1}\left|\dot{x}_{a v g}-\dot{x}_{t g t}\right|+c_{2} C_{E}
$$

where $\dot{x}_{a v g}$ and $\dot{x}_{t g t}$ are average and target walking speeds, and $C_{E}$ is the energetic cost. The parameters $c_{1}$ and $c_{2}$ are empirically determined constant coefficients, 10 and 1 , respectively. $\dot{x}_{t g t}$ is set to normal walking speed $1.4 \mathrm{~ms}^{-1}$, and $\dot{x}_{a v g}$ and $C_{E}$ are computed during multiple consecutive steps of steady walking. We define a step as a steady step if the positions of edges of every segments do not change significantly relative to the touch-down position between subsequent heel-strikes. Specifically, we define $d_{\text {steady }} \leq$ $3 \mathrm{~cm}$ as a criterion for a steady step, where $d_{\text {steady }}$ is the sum of the differences of the relative positions. Through extensive simulations, six consecutive steady steps proved to be a reliable indicator of steady walking. We compute the energetic cost as $C_{E}=E_{M} /\left(m x_{d}\right)$, where $E_{M}$ is the total metabolic energy consumed by all muscles estimated from a muscle energy model [18], $m$ is the body mass, and $x_{d}$ is the walking distance traveled.

\section{Simulation Setup}

We optimize the control parameters for six values of $k_{M T J}\left(250,500,1000,2000,4000, \infty \mathrm{Nmrad}^{-1}\right.$, where $\infty \mathrm{Nmrad}^{-1}$ indicates a rigid foot). The minimum value $250 \mathrm{Nmrad}^{-1}$ was chosen to prevent the foot from collapsing during walking, and we did not explore for stiffness values between $4000 \sim \infty \mathrm{Nmrad}^{-1}$, since the foot with $4000 \mathrm{Nmrad}^{-1}$ showed little deformation during walking and its energy cost did not show much difference from that of $2000 \mathrm{Nmrad}^{-1}$. To verify the optimization results for each $k_{M T J}$ value, we used the optimized parameter values as initial values and repeated the optimization until CMA-ES does not find better parameters.

\section{HUMAN SUBJECT EXPERIMENT WITH COMPLIANT FEET}

\section{A. Hardware Design of the Compliant Foot}

For the experimental test on the effect of foot compliance on energy efficiency, we developed a compliant shoe (Fig. 3b). The shoe was designed to be able to easily change the stiffness values while conducting experiments. Specifically, a linear spring was mounted on a piece that can slide back and forth which changes the effective moment arm. The design allows a test range for the midtarsal joint stiffness from $500 \mathrm{Nmrad}^{-1}$ to $2200 \mathrm{Nmrad}^{-1}$, which in simulation showed a large energetic difference (gray region in Fig. 4a). A second linear spring was added at the heel to mimic the heel pad in humans. Both springs connected to a plate which had a bicycle shoe mounted. The shoe had a stiff sole to isolate internal foot motions from studying the artificially imposed windlass mechanism. The mass of the device including the bicycle shoe was about $0.9 \mathrm{~kg}$ and the distance from the ground to the foot sole was about $7 \mathrm{~cm}$. 
(a)

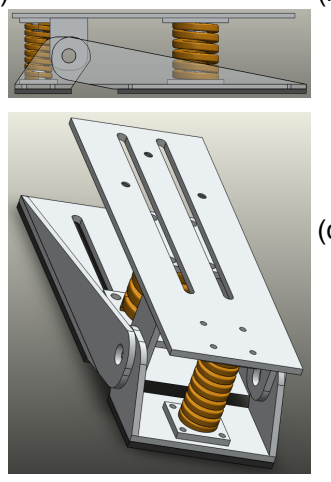

(b)

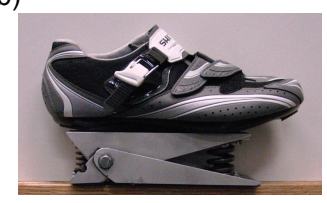

(d)

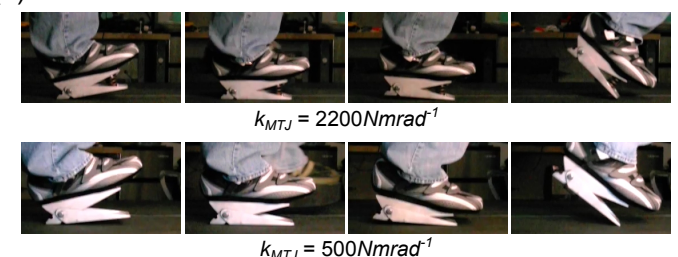

(c)

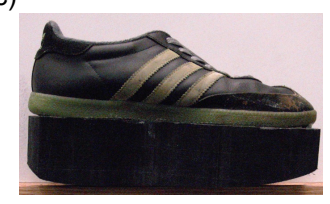

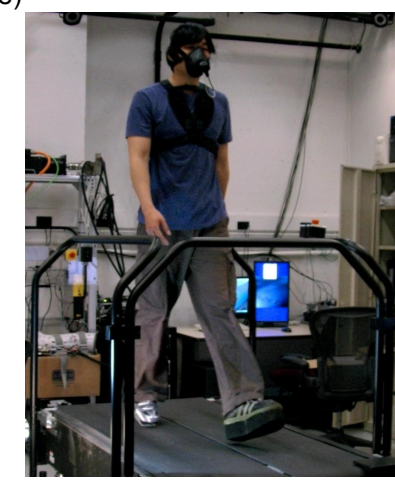

Fig. 3. Human subject experiment. (a) A CAD drawing of the lower part of the compliant shoe. (b) Side view of the compliant shoe. The value of $k_{M T J}$ can be adjusted by sliding and fixing the front spring at different locations. The ground contact surface is about $20 \mathrm{~cm} \times 6 \mathrm{~cm}$ and the total mass of the compliant shoe is about $0.9 \mathrm{~kg}$. With the compliant shoe on, the foot height becomes about $7 \mathrm{~cm}$. To match this height, the subject wears a second, elevated shoe (c) which weighs about $0.7 \mathrm{~kg}$. (d) Deflection of the compliant shoe at extreme values of $k_{M T J}$ during walking. (e) Subject walking on the treadmill wearing the compliant and elevated shoes with a respirometry system to measure the metabolic cost.

\section{B. Experimental Setup}

We compared the metabolic energy consumptions during human walking trials wearing the compliant shoe with three different $k_{M T J}$ values ranging from soft to stiff $(500,1300$ and $2200 \mathrm{Nmrad}^{-1}$ ). The experiments were conducted with two male adult subjects (subject1: $178 \mathrm{~cm}, 68 \mathrm{~kg}$; subject2: $182 \mathrm{~cm}, 78 \mathrm{~kg}$ ) over a period of two days. On the first day, the subjects trained walking with the compliant shoe for five minutes with all three $k_{M T J}$ values to get used to the compliant foot device. On the next day, the metabolic costs were measured during the last three minutes of fiveminute walking trials, with ten-minute rests between the trials. In the trials, the subjects walked on a treadmill at normal walking speed $\left(1.3 \mathrm{~ms}^{-1}\right)$, with the compliant shoe on the right foot and an elevated shoe on the left foot, matching the lengths of both legs (Fig. 3-c,e). The metabolic rate $\dot{E}[W]=16.46 \dot{V}_{\mathrm{O} 2}\left[\mathrm{ml} \mathrm{s}^{-1}\right]+4.48 \dot{V}_{\mathrm{CO} 2}\left[\mathrm{ml} \mathrm{s}^{-1}\right]$ was estimated from the rate of oxygen consumption $\dot{V}_{O 2}$ and carbon dioxide production $\dot{V}_{\mathrm{CO} 2}$ measured with a respirometry system (Oxycon Mobile, JAEGER [19]). The energetic cost was then calculated as $C_{E}=\left(\dot{E}_{\text {walk }}-\dot{E}_{\text {stand }}\right) /(m v)$, where $\dot{E}_{\text {walk }}$ and $\dot{E}_{\text {stand }}$ are the metabolic rates measured during walking and quiet-standing, and $m$ and $v$ are the subject's mass and walking speed.

\section{PRELIMINARY RESULTS}

For each value of $k_{M T J}$, the optimized energetic costs of simulated walking are presented in Fig. 4-a. The identified $C_{E}$ values range from 2.2 to $2.4 \mathrm{Jkg}^{-1} \mathrm{~m}^{-1}$. The experimental observed values of the energetic cost of 2.1 to $2.3 \mathrm{Jkg}^{-1} \mathrm{~m}^{-1}$ fall within a similar range. The optimization results further suggest that softer values of $k_{M T J}$ incur more energetic cost during walking, and the preliminary experimental results confirm this trend (Fig. 4-b). The difference between walking with the softest and the stiffest setting is about $7 \%$ and $10 \%$ of the total energetic cost for each subject.

\section{CONCLUSION AND FUTURE DIRECTIONS}

The results from the simulation and experimental studies so far suggest that the energy saved by the windlass mechanism [14] does not originate from the compliance it embeds in the foot. The results actually indicate that stiff feet generate more energy effective locomotion than soft ones. A possible explanation for this observation is that the power transfer of the leg to the ground is more effective with stiff feet. Given our initial hypothesis that the energy saving generated by the windlass mechanism [14] comes either from the foot compliance or from the mechanism's property of reducing the effective foot length in swing, the results suggest that the second option is more likely.

In future work, we plan to better understand why compliant feet decrease the energy efficiency of walking, for instance, by comparing the energetic costs at the individual muscle and joint levels. In addition, we plan to investigate in simulation and experimental studies the effect of the foot length reduction on the energetic efficiency of human walking, testing the second option of our original hypothesis. Although some studies on the effect of foot length on energetic cost exist [20], [21], these studies used completely immobilized ankle-foot complexes, which makes it difficult to extract the effect of the windlass mechanism as it does not impede the motion of the ankle.

\section{ACKNOWLEDGMENT}

The authors thank J. Caputo for helping on conducting the human experiments.

\section{REFERENCES}

[1] (2013, Jan.) össur americas. [Online]. Available: http://www.ossur.com/americas

[2] (2013, Dec.) iwalk. the iwalk prosthetic foot and ankle addresses amputee clinical problems. [Online]. Available: http://www.iwalkpro.com/

[3] $(2013$, Jan.) Ottobock global start. [Online]. Available: http://www.ottobock.com/ 
(a) Simulation

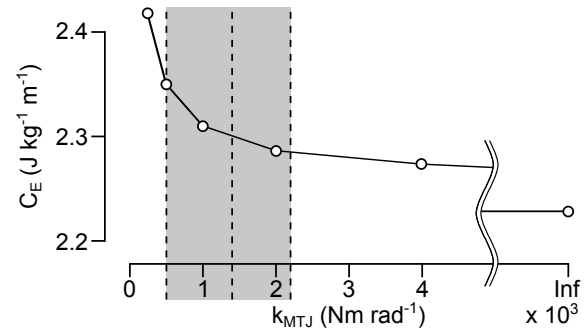

(b) Experiment

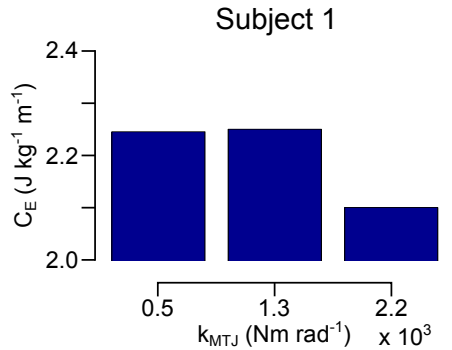

Subject 2

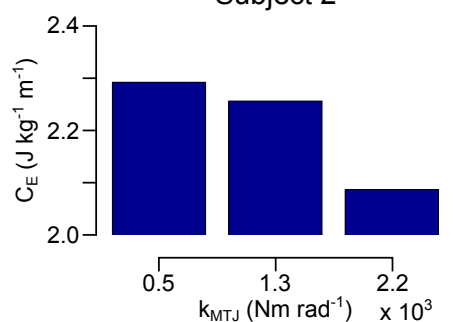

Fig. 4. Results of the simulation and experimental studies. (a) Minimized energetic costs for each value of $k_{M T J}$. The shaded area indicates the range of $k_{M T J}$ that the hardware compliant shoe covers, and the dotted lines correspond to the $k_{M T J}$ values used in the human subjects experiments. (b) Preliminary results for two subjects.

[4] M. Eilenberg, H. Geyer, and H. Herr, "Control of a powered anklefoot prosthesis based on a neuromuscular model," IEEE Trans Neural Syst Rehabil Eng, vol. 18, no. 2, pp. 164-173, 2010.

[5] S. Collins and A. Kuo, "Recycling energy to restore impaired ankle function during human walking," PloS one, vol. 5, no. 2, p. e9307, 2010.

[6] F. Sup, H. Varol, and M. Goldfard, "Upslope walking with a powered knee and ankle prosthesis: Intitial results with an amputee subject," IEEE/ASME Trans. Neural. Syst. Rehabil. Eng., vol. 19, no. 1, pp. 71-78, 2011.

[7] J. H. Hicks, "The mechanics of the foot - i. the joints," J. Anat., vol. 87, no. 4, pp. 345-357, 1954.

[8] S. Scott, D. Winter, et al., "Biomechanical model of the human foot: kinematics and kinetics during the stance phase of walking." Journal of biomechanics, vol. 26, no. 9, p. 1091, 1993.

[9] F. Bojsen-Moller and K. E. Flagstad, "Plantar aponeurosis and internal architecture of the ball of the foot," J. Anat., vol. 121, no. 3, pp. 599611, 1976.

[10] P. J. Briggs and P. A. Tansey, "Active and passive mechanisms in the control of supination," Foot Ankle Surg., vol. 7, pp. 131-136, 2001.

[11] J. H. Hicks, "The mechanics of the foot - ii. the plantar aponeurosis and the arch," J. Anat., vol. 88, no. 1, pp. 25-30, 1954.

[12] A. Kappel-Bargas, R. Woolf, M. Cornwall, and T. Mcpoil, "The windlass mechanism during normal walking and passive first metatarsalphalangeal joint extension," Clinical Biomechanics, vol. 13, no. 3, pp. 190-194, 1998.

[13] E. Fuller, "The windlass mechanism of the foot - a mechanical model to explain pathology," Journal of the American Podiatric Medical Association, vol. 90, no. 1, pp. 35-46, 2000.

[14] S. Song and H. Geyer, "The energetic cost of adaptive feet in walking," in Robotics and Biomimetics (ROBIO), 2011 IEEE International Conference on. IEEE, 2011, pp. 1597-1602.

[15] H. Geyer and H. M. Herr, "A muscle-reflex model that encodes principles of legged mechanics produces human walking dynamics and muscle activities," IEEE Trans. Neural Syst. Rehab. Eng., vol. 18, no. 3, 2010.

[16] N. Hansen, "The cma evolution strategy: A comparing review," Towards a New Evolutionary Computation. Advances on Estimation of Distribution Algorithms. Springer, p. 75.102, 2006.

[17] S. Song and H. Geyer, "Regulating speed and generating large speed transitions in a neuromuscular human walking model," in Robotics and Automation (ICRA), 2012 IEEE International Conference on. IEEE, 2012, pp. 511-516.

[18] B. R. Umberger, K. G. Gerritsen, and P. E. Martin, "A model of human muscle energy expenditure," Comp. Meth. Biomech. Biomedic. Eng., vol. 6, no. 2, pp. 99-111, 2003.

[19] (2013, Jan.) Carefusion, oxycon moblie - carefusion. [Online]. Available: http://www.carefusion.com.au

[20] P. Adamczyk, S. Collins, and A. Kuo, "The advantages of a rolling foot in human walking," Journal of Experimental Biology, vol. 209, no. 20, pp. 3953-3963, 2006.

[21] T.-w. Huang, K. E. Zelik, P. G. Adamczyk, and A. D. Kuo, "Effect of foot length on walking with a compliant foot," in Gait and Clinical Movement Analysis Society, 2012. 